\title{
Incomplete character sums over finite fields and their application to the interpolation of the discrete logarithm by Boolean functions
}

by

Tanja Lange (Essen) and Arne Winterhof (Wien)

1. Introduction. Let $\mathbb{F}_{q}$ denote the finite field of order $q=p^{r}$ with a prime $p$ and an integer $r \geq 1$. Let $\left\{\beta_{0}, \ldots, \beta_{r-1}\right\}$ be a basis of $\mathbb{F}_{q}$ over $\mathbb{F}_{p}$ and define $\xi_{k}$ for $0 \leq k<q$ by

$$
\xi_{k}=k_{0} \beta_{0}+k_{1} \beta_{1}+\ldots+k_{r-1} \beta_{r-1}
$$

if

$$
k=k_{0}+k_{1} p+\ldots+k_{r-1} p^{r-1} \quad \text { with } 0 \leq k_{i}<p \text { for } 0 \leq i<r .
$$

For $1 \leq K \leq p$ put

$$
\mathcal{K}_{K}=\left\{k=k_{0}+k_{1} p+\ldots+k_{r-1} p^{r-1} \mid 0 \leq k_{i}<K \text { for } 0 \leq i<r\right\} .
$$

Consider the incomplete character sums $\sum_{k \in \mathcal{K}_{K}} \chi\left(f\left(\xi_{k}\right)\right)$, where $f \in \mathbb{F}_{q}[x]$ and $\chi$ is a multiplicative character of $\mathbb{F}_{q}$. Excluding trivial cases we show in Section 2 that these sums are at most of the order of magnitude

$$
O\left(K^{1 / 2} p^{1 / 4}\right) \quad \text { if } r=1 \quad \text { and } \quad O\left(K^{r-1} p^{1 / 2}\right) \quad \text { if } r \geq 2,
$$

which improves previous results obtained with the standard method of Pólya and Vinogradov for $K$ of the order of magnitude between $O\left(p^{1 / 2}\right)$ and $O\left(p^{1 / 2}(\log (p))^{2}\right)$ if $r=1$ and $O\left(p^{1 / 2}(\log (p))^{r /(r-1)}\right)$ if $r \geq 2$.

If $\gamma$ is a primitive element of $\mathbb{F}_{q}$ and $\xi \in \mathbb{F}_{q}, \xi \neq 0$, then $\xi=\gamma^{l}$ for some integer $l$ with $0 \leq l \leq q-2$ and we say that $l$ is the discrete logarithm (or index) of $\xi$ to the base $\gamma$, denoted by $\operatorname{ind}_{\gamma}(\xi)=l$. For many practical purposes it would be sufficient to have an easily computable function which represents $\operatorname{ind}_{\gamma}(\xi)$ for almost all $\xi \neq 0$ or at least its rightmost bit, which is obviously 0 if $\xi$ is a square in $\mathbb{F}_{q}$ and 1 if $\xi$ is a non-square in $\mathbb{F}_{q}$ in the case of $p>2$. To obtain a lower bound on the complexity of the discrete logarithm we investigate interpolating Boolean functions.

2000 Mathematics Subject Classification: 11T23, 11T71, 11Y16, 94A60, 94C10. 
A Boolean function $B$ can be represented as a multilinear polynomial over $\mathbb{F}_{2}$ and the sparsity (or weight) $\operatorname{spr}(B)$ of $B$ is the number of non-zero coefficients of $B$. In the special case when $r=1$ and $\beta_{0}=1$, i.e. $\xi_{k}=k$ for $0 \leq k<p$, and $p>2$, in Coppersmith and Shparlinski [1, Theorem 5] and Shparlinski [10, Theorem 6.1] it was shown that for a Boolean function $B\left(U_{1}, \ldots, U_{s}\right)$ of $s=\left\lfloor\log _{2}(p)\right\rfloor$ variables satisfying

$$
B\left(u_{1}, \ldots, u_{s}\right)= \begin{cases}0 & \text { if } k \text { is a quadratic residue in } \mathbb{F}_{p}, \\ 1 & \text { if } k \text { is a quadratic non-residue in } \mathbb{F}_{p},\end{cases}
$$

where $k=u_{1}+\ldots+u_{s} 2^{s-1}$ with $u_{j} \in\{0,1\}$ for $1 \leq j \leq s$ and $1 \leq k<2^{s}$, we have

$$
\operatorname{spr}(B) \geq 2^{-3 / 2} p^{1 / 4}\left(\log _{2}(p)\right)^{-1 / 2}-1 .
$$

Shparlinski mentioned in $[10$, p. 145] that using a "symmetrization" trick one can replace $p^{1 / 4}\left(\log _{2}(p)\right)^{-1 / 2}$ by $p^{1 / 4}$ in (2) with a slightly worse constant. In Section 3 we extend the latter result to arbitrary $r$. The proof is based on the new estimate (1) for incomplete character sums.

2. A bound for incomplete character sums. Let $\chi$ be a non-trivial multiplicative character of $\mathbb{F}_{q}$ of order $t$, with the convention $\chi(0)=0$, and let $f(x) \in \mathbb{F}_{q}[x]$ be a monic polynomial of positive degree that is not a $t$ th power of a polynomial. Let $v$ be the number of distinct roots of $f(x)$ in its splitting field over $\mathbb{F}_{q}$. First we recall Weil's bound for complete character sums.

LEMma 1. We have

$$
\left|\sum_{\xi \in \mathbb{F}_{q}} \chi(f(\xi))\right| \leq(v-1) q^{1 / 2}
$$

Proof. Lidl and Niederreiter [4, Theorem 5.41].

Now we prove a new bound for incomplete character sums.

Theorem 1. For $1 \leq K<p$ we have

$$
\left|\sum_{k \in \mathcal{K}_{K}} \chi\left(f\left(\xi_{k}\right)\right)\right|<K^{r / 2}(3 v-1)^{1 / 2} q^{1 / 4}+r p^{1 / 2} K^{r-1} .
$$

Proof. We modify the method used in Niederreiter and Shparlinski [7]. (See also Gutierrez, Niederreiter, and Shparlinski [3], Niederreiter and Shparlinski [6], and Niederreiter and Winterhof [8].) For any integer

$$
m=m_{0}+m_{1} p+\ldots+m_{r-1} p^{r-1} \quad \text { with } 0 \leq m_{i}<p \text { for } 0 \leq i<r
$$

we have

$$
\left|\sum_{k \in \mathcal{K}_{K}} \chi\left(f\left(\xi_{k}\right)\right)-\sum_{k \in \mathcal{K}_{K}} \chi\left(f\left(\xi_{k}+\xi_{m}\right)\right)\right| \leq 2\left(m_{0}+\ldots+m_{r-1}\right) K^{r-1} .
$$


(We have $\xi_{k}+\xi_{m} \neq \xi_{l}$ for all $l \in \mathcal{K}_{K}$ only if at least one coordinate $k_{i}$ of $\xi_{k}$ satisfies $k_{i}+m_{i} \geq K$. The number of possible $k \in \mathcal{K}_{K}$ with this property is at most $\left(m_{0}+\ldots+m_{r-1}\right) K^{r-1}$. Similarly we can verify that the number of $k$ with $\xi_{k} \neq \xi_{l}+\xi_{m}$ for all $l \in \mathcal{K}_{K}$ is at most $\left(m_{0}+\ldots+m_{r-1}\right) K^{r-1}$.) Then for any integer $M$ with $1 \leq M \leq p$ we have

$$
2 \sum_{m \in \mathcal{K}_{M}}\left(m_{0}+\ldots+m_{r-1}\right)=r M^{r}(M-1)
$$

and

$$
M^{r}\left|\sum_{k \in \mathcal{K}_{K}} \chi\left(f\left(\xi_{k}\right)\right)\right| \leq W+r M^{r}(M-1) K^{r-1},
$$

where

$$
W=\left|\sum_{k \in \mathcal{K}_{K}} \sum_{m \in \mathcal{K}_{M}} \chi\left(f\left(\xi_{k}+\xi_{m}\right)\right)\right| \leq \sum_{k \in \mathcal{K}_{K}}\left|\sum_{m \in \mathcal{K}_{M}} \chi\left(f\left(\xi_{k}+\xi_{m}\right)\right)\right| .
$$

Using the Cauchy-Schwarz inequality we obtain

$$
\begin{aligned}
W^{2} & \leq K^{r} \sum_{k \in \mathcal{K}_{K}}\left|\sum_{m \in \mathcal{K}_{M}} \chi\left(f\left(\xi_{k}+\xi_{m}\right)\right)\right|^{2} \leq K^{r} \sum_{\xi \in \mathbb{F}_{q}}\left|\sum_{m \in \mathcal{K}_{M}} \chi\left(f\left(\xi+\xi_{m}\right)\right)\right|^{2} \\
& =K^{r} \sum_{m, m^{\prime} \in \mathcal{K}_{M}} \sum_{\xi \in \mathbb{F}_{q}} \chi\left(f\left(\xi+\xi_{m}\right) f\left(\xi+\xi_{m^{\prime}}\right)^{t-1}\right) .
\end{aligned}
$$

Let $f(x)=\prod_{j=1}^{v}\left(x-\nu_{j}\right)^{c_{j}}$ be the factorization of $f(x)$ in its splitting field. Since $f(x)$ is not a $t$ th power, there exists some $h$ with $1 \leq h \leq v$ and $c_{h} \not \equiv 0 \bmod t$. If

$$
\xi_{m}=\xi_{m^{\prime}}+\nu_{h}-\nu_{j} \quad \text { for some } j \text { with } 1 \leq j \leq v,
$$

then the sum over $\xi$ is estimated trivially by $q$. (There are at most $v$ possible indices $m^{\prime}$ satisfying (4) for given $m$ and $h$.) If $\xi_{m} \neq \xi_{m^{\prime}}+\nu_{h}-\nu_{j}$ for all $j$ with $1 \leq j \leq v$, then the polynomial $g(x)=f\left(x+\xi_{m}\right) f\left(x+\xi_{m^{\prime}}\right)^{t-1}$ is not a $t$ th power and has at most $2 v$ distinct zeros. Hence,

$$
W^{2} \leq K^{r} M^{r} v q+K^{r} M^{2 r}(2 v-1) q^{1 / 2}
$$

by Lemma 1 . Choosing $M=\left\lceil p^{1 / 2}\right\rceil$ we get

$$
W^{2} / M^{2 r}<K^{r}(3 v-1) q^{1 / 2}
$$

and the assertion by (3).

Corollary 1. For $1 \leq K<p$ we have

$$
\left|\sum_{k \in \mathcal{K}_{K}} \chi\left(f\left(\xi_{k}\right)\right)\right|< \begin{cases}2.2 K^{1 / 2} v^{1 / 2} p^{1 / 4} & \text { if } r=1 \\ \left(3^{1 / r}+r\right) K^{r-1} v^{1 / r} p^{1 / 2} & \text { if } r \geq 2\end{cases}
$$

Proof. Since otherwise the bound is trivial we may assume that either $r=1$ and $K \geq 4.84 p^{1 / 2}$ or $r \geq 2$ and $K \geq 3^{1 / r} v^{1 / r} p^{1 / 2}$. Then Theorem 1 
yields for $r=1$,

$$
\left|\sum_{k \in \mathcal{K}_{K}} \chi\left(f\left(\xi_{k}\right)\right)\right|<K^{1 / 2} v^{1 / 2} p^{1 / 4}\left(\sqrt{3}+K^{-1 / 2} p^{1 / 4}\right)<2.2 K^{1 / 2} v^{1 / 2} p^{1 / 4},
$$

and for $r \geq 2$,

$$
\begin{aligned}
\left|\sum_{k \in \mathcal{K}_{K}} \chi\left(f\left(\xi_{k}\right)\right)\right| & <K^{r-1} v^{1 / r} p^{1 / 2}\left(3^{1 / 2} K^{-r / 2+1} v^{1 / 2-1 / r} p^{r / 4-1 / 2}+r\right) \\
& \leq\left(3^{1 / r}+r\right) K^{r-1} v^{1 / r} p^{1 / 2}
\end{aligned}
$$

which completes the proof.

Remarks. 1. The standard method of Pólya and Vinogradov yields

$$
\left|\sum_{k \in \mathcal{K}_{K}} \chi\left(f\left(\xi_{k}\right)\right)\right|<v q^{1 / 2}(1+\log (p))^{r}
$$

(see Davenport and Lewis [2, Theorem 1] for linear polynomials and Winterhof [11, Theorem 2] for arbitrary polynomials). Equation (5) is only nontrivial if $K$ is at least of the order of magnitude $O\left(p^{1 / 2} \log (p)\right)$. Theorem 1 is non-trivial if $K$ is at least of the order of magnitude $O\left(p^{1 / 2}\right)$ and it is better than (5) if $K$ is at most of the order of magnitude $O\left(p^{1 / 2}(\log (p))^{2}\right)$ if $r=1$ and $O\left(p^{1 / 2}(\log (p))^{r /(r-1)}\right)$ if $r \geq 2$.

2. In [9, Theorem 3.1] Niederreiter and the second author showed that for any $1 \leq K<q$ we have

$$
\left|\sum_{k=0}^{K-1} \chi\left(f\left(\xi_{k}\right)\right)\right|<K^{1 / 2}(3 v-1)^{1 / 2} q^{1 / 4}+q^{1 / 2} .
$$

For $r=1$ Theorem 1 and (6) coincide.

3. Interpolation by Boolean functions. In this section we give lower bounds for the sparsity and the degree of a Boolean function representing the rightmost bit of the discrete logarithm for almost all non-zero elements of $\mathbb{F}_{q}$.

Theorem 2. Let $p>2$. Put $s=\left\lfloor\log _{2}(p)\right\rfloor$, and let

$$
B\left(U_{11}, \ldots, U_{1 s}, \ldots, U_{r 1}, \ldots, U_{r s}\right)
$$

be a Boolean function satisfying

$$
B\left(u_{11}, \ldots, u_{1 s}, \ldots, u_{r 1}, \ldots, u_{r s}\right)= \begin{cases}0 & \text { if } \xi_{k} \text { is a square in } \mathbb{F}_{q}, \\ 1 & \text { if } \xi_{k} \text { is a non-square in } \mathbb{F}_{q},\end{cases}
$$

where $k_{i-1}=u_{i 1}+u_{i 2} 2+\ldots+u_{i s} 2^{s-1}$ with $u_{i j} \in\{0,1\}$ for $1 \leq j \leq s$, $1 \leq i \leq r$, and $k \in \mathcal{K}_{2^{s}} \backslash\{0\}$. Then $\operatorname{spr}(B)$ is at least of the order of magnitude $O\left(q^{1 / 4}\right)$, where the implied constant depends only on $r$. 
Proof. Define the integer $a$ by $2^{a}>(\operatorname{spr}(B)+1)^{1 / r} \geq 2^{a-1}$ and put $\mathcal{M}=\left\{0, \ldots, 2^{a}-1\right\}^{r} \backslash\{(0, \ldots, 0)\}$. For each $\underline{m}=\left(m_{1}, \ldots, m_{r}\right) \in \mathcal{M}$ we consider the function

$$
\begin{aligned}
& B_{\underline{m}}\left(U_{11}, \ldots, U_{1, s-a}, \ldots, U_{r 1}, \ldots, U_{r, s-a}\right) \\
& \quad:=B\left(U_{11}, \ldots, U_{1, s-a}, m_{11}, \ldots, m_{1 a}, \ldots, U_{r 1}, \ldots, U_{r, s-a}, m_{r 1}, \ldots, m_{r a}\right),
\end{aligned}
$$

where $m_{i}=m_{i 1}+\ldots+m_{i a} 2^{a-1}$ with $m_{i j} \in\{0,1\}$ for $1 \leq j \leq a$ and $1 \leq i \leq r$.

The number of distinct monomials in $U_{11}, \ldots, U_{1, s-a}, \ldots, U_{r 1}, \ldots, U_{r, s-a}$ occurring in all the $B_{\underline{m}}$ does not exceed $\operatorname{spr}(B)$. Since $|\mathcal{M}|=2^{\text {ar }}-1>$ $\operatorname{spr}(B)$ we can find a non-trivial linear combination

$$
\sum_{\underline{m} \in \mathcal{M}} c_{\underline{m}} B_{\underline{m}}\left(U_{11}, \ldots, U_{1, s-a}, \ldots, U_{r 1}, \ldots, U_{r, s-a}\right) \quad \text { with } c_{\underline{m}} \in \mathbb{F}_{2} \text { for } \underline{m} \in \mathcal{M}
$$

which vanishes identically.

Let $\chi$ be the quadratic character of $\mathbb{F}_{q}$. By the condition of the theorem we have

$$
\chi\left(\xi_{k}\right)=(-1)^{B\left(u_{11}, \ldots, u_{1 s}, \ldots, u_{r 1}, \ldots, u_{r s}\right)} \quad \text { for } k \in \mathcal{K}_{2^{s}} \backslash\{0\} .
$$

Put $K=2^{s-a}$. Then for $k=k_{0}+k_{1} p+\ldots+k_{r-1} p^{r-1} \in \mathcal{K}_{K}$ we have

$$
\begin{aligned}
\prod_{\underline{m} \in \mathcal{M}} \chi\left(\left(k_{0}+m_{1} 2^{s-a}\right) \beta_{0}+\ldots+\left(k_{r-1}+m_{r} 2^{s-a}\right) \beta_{r-1}\right)^{c_{\underline{m}}} \\
=(-1)^{\sum_{\underline{m} \in \mathcal{M}} c_{\underline{m}} B_{\underline{m}}\left(u_{11}, \ldots, u_{1, s-a}, \ldots, u_{r 1}, \ldots, u_{r, s-a}\right)}=1
\end{aligned}
$$

and thus

$$
2^{(s-a) r}=\sum_{k \in \mathcal{K}_{K}} \chi\left(\prod_{\underline{m} \in \mathcal{M}}\left(\left(k_{0}+m_{1} 2^{s-a}\right) \beta_{0}+\ldots+\left(k_{r-1}+m_{r} 2^{s-a}\right) \beta_{r-1}\right)^{c_{\underline{m}}}\right) .
$$

Hence, for $r=1$ Corollary 1 yields

$$
2^{s-a}<2.2 \cdot 2^{s / 2} p^{1 / 4}
$$

and thus

$$
2^{a}>0.45 \cdot 2^{s / 2} p^{-1 / 4} \geq 0.31 p^{1 / 4} .
$$

Hence,

$$
\operatorname{spr}(B) \geq 2^{a-1}-1>0.15 p^{1 / 4}-1 .
$$

For $r \geq 2$ Corollary 1 yields

$$
2^{(s-a) r}<\left(3^{1 / r}+r\right) 2^{(s-a)(r-1)} 2^{a} p^{1 / 2} .
$$

Hence,

$$
2^{2 a}>\left(3^{1 / r}+r\right)^{-1} p^{-1 / 2} 2^{s} \geq 2^{-1}\left(3^{1 / r}+r\right)^{-1} p^{1 / 2}
$$

and thus

$$
(\operatorname{spr}(B)+1)^{1 / r} \geq 2^{a-1} \geq 2^{-3 / 2}\left(3^{1 / r}+r\right)^{-1 / 2} p^{1 / 4},
$$

which yields the assertion. 
Using this bound we obtain the following bound on the degree of the Boolean function $B$.

Corollary 2. Under the conditions of Theorem 2 for any $r \geq 1$ and any $\varepsilon>0$ there exists a $p_{0}(\varepsilon, r)$ such that for all $p \geq p_{0}$ we have

$$
\operatorname{deg}(B)>(0.04-\varepsilon) r s .
$$

Proof. Put $n=\operatorname{deg}(B)$. Since otherwise the corollary is trivial we may suppose $2 n \leq r s$. Obviously,

$$
\operatorname{spr}(B) \leq \sum_{i=0}^{n}\left(\begin{array}{c}
r s \\
i
\end{array}\right) \leq 2^{r s H(n /(r s))}
$$

by van Lint [5, Theorem 1.4.5], where $H(x)=-x \log _{2}(x)-(1-x) \log _{2}(1-x)$ for $0<x \leq 1 / 2$ denotes the binary entropy function. Equations (7) and (8) yield

$$
H\left(\frac{n}{r s}\right) \geq \frac{1}{4}+\frac{c}{s}
$$

with a constant $c<0$ depending only on $r$ and thus

$$
n \geq(\theta-\varepsilon) r s \quad \text { for } p \geq p_{0},
$$

where $\theta>0.04$ denotes the solution of $H(x)=1 / 4$.

Remarks. 1. Theorem 2 and Corollary 2 can be improved if 2 is a nonsquare in $\mathbb{F}_{q}$, i.e. if and only if $q \equiv \pm 3 \bmod 8$. Then we define $F\left(U_{11}, \ldots\right.$, $\left.U_{1, s-1}, \ldots, U_{r 1}, \ldots, U_{r, s-1}\right)$ by

$$
\begin{aligned}
F\left(U_{11}, \ldots, U_{1, s-1}, \ldots,\right. & \left.U_{r 1}, \ldots, U_{r, s-1}\right) \\
:= & B\left(U_{11}, \ldots, U_{1, s-1}, 0, \ldots, U_{r 1}, \ldots, U_{r, s-1}, 0\right) \\
& +B\left(0, U_{11}, \ldots, U_{1, s-1}, \ldots, 0, U_{r 1}, \ldots, U_{r, s-1}\right) .
\end{aligned}
$$

We have $F\left(u_{11}, \ldots, u_{1, s-1}, \ldots, u_{r 1}, \ldots, u_{r, s-1}\right)=1$ for every non-zero $\xi_{k}$ with $k \in \mathcal{K}_{2^{s-1}}$ since exactly one of $\xi_{k}$ and $2 \xi_{k}$ is a square in $\mathbb{F}_{q}$. With $F(0, \ldots, 0)=0$ (which does not depend on the ambiguous value of $B(0, \ldots, 0))$ we get

$$
F\left(U_{11}, \ldots, U_{r, s-1}\right)=\prod_{\substack{1 \leq i \leq r \\ 1 \leq j \leq s-1}}\left(1+U_{i j}\right)+1 .
$$

From the definition of $F$ we have

$$
\operatorname{deg}(B) \geq \operatorname{deg}(F)=r(s-1)
$$

and

$$
\operatorname{spr}(B) \geq\lceil 0.5 \operatorname{spr}(F)\rceil=\left\lceil 0.5\left(2^{r(s-1)}-1\right)\right\rceil=2^{r(s-1)-1} \geq \frac{q}{2^{2 r+1}} .
$$

For $r=1$ these results were derived by Shparlinski [10, Section 6]. 
2. In the same way as in the proof of Shparlinski [10, Theorem 6.2] one can use Corollary 2 to deduce a lower bound for the depth $d$ of bounded fan-in Boolean circuits representing the rightmost bit of $\operatorname{ind}_{\gamma}\left(\xi_{k}\right)$ for all $k \in \mathcal{K}_{2^{s}} \backslash\{0\}$ in case of arbitrary $r$ :

$$
d \geq \log _{2}(r s)+O(1)
$$

Acknowledgments. This paper was written during a visit of the first author to the Austrian Academy of Sciences. She wishes to thank Prof. $\mathrm{H}$. Niederreiter and the Institute of Discrete Mathematics for hospitality and financial support.

\section{References}

[1] D. Coppersmith and I. E. Shparlinski, On polynomial approximation of the discrete logarithm and the Diffie-Hellman mapping, J. Cryptology 13 (2000), 339-360.

[2] H. Davenport and D. J. Lewis, Character sums and primitive roots in finite fields, Rend. Circ. Mat. Palermo (2) 12 (1963), 129-136.

[3] J. Gutierrez, H. Niederreiter and I. E. Shparlinski, On the multidimensional distribution of inversive congruential pseudorandom numbers in parts of the period, Monatsh. Math. 129 (2000), 31-36.

[4] R. Lidl and H. Niederreiter, Finite Fields, Cambridge Univ. Press, Cambridge, 1997.

[5] J. H. van Lint, Introduction to Coding Theory, Springer, New York, 1982.

[6] H. Niederreiter and I. E. Shparlinski, Exponential sums and the distribution of inversive congruential pseudorandom numbers with prime-power modulus, Acta Arith. 92 (2000), 89-98.

[7] - - - On the distribution of inversive congruential pseudorandom numbers in parts of the period, Math. Comp. 70 (2001), 1569-1574.

[8] H. Niederreiter and A. Winterhof, Incomplete exponential sums over finite fields and their applications to new inversive pseudorandom number generators, Acta Arith. 93 (2000), 387-399.

[9] - - - Incomplete character sums over finite fields and polynomial interpolation of the discrete logarithm, Finite Fields Appl., to appear.

[10] I. E. Shparlinski, Number Theoretic Methods in Cryptography: Complexity Lower Bounds, Birkhäuser, Basel, 1999.

[11] A. Winterhof, Some estimates for character sums and applications, Des. Codes Cryptogr. 22 (2001), 123-131.

Institute of Experimental Mathematics

University of Essen

Ellernstraße 29

D-45326 Essen, Germany

E-mail: lange@exp-math.uni-essen.de
Institute of Discrete Mathematics

Austrian Academy of Sciences

Sonnenfelsgasse 19

A-1010 Wien, Austria

E-mail: arne.winterhof@oeaw.ac.at

Received on 3.8.2000

and in revised form on 17.5.2001 